OAI-PMH: http://www.indteca.com/ojs/index.php/Revista Scientific/oai

Artículo Original / Original Article

\title{
Experiencias Lúdicas en el Desarrollo del Pensamiento Lógico
}

\author{
Autora: María Nazareth Rocca Báez \\ Universidad Pedagógica Experimental Libertador, UPEL \\ nazarethrocca2017@gmail.com \\ Barinas, Venezuela \\ https://orcid.org/0000-0003-4160-0283
}

\section{Resumen}

La investigación tuvo como propósito implementar experiencias lúdicas para la enseñanza del pensamiento lógico en el en Centro de Educación Inicial Bolivariano Misia Jacinta de Crespo, parroquia Corazón de Jesús del municipio Barinas, estado Barinas. Metodológicamente, se sustentó en el paradigma socio crítico bajo el método de investigación acción en las fases aportadas por Hurtado y Toro (2005), diagnóstico, planificación, ejecución y evaluación; siendo los informantes dos (2) docentes, dieciséis (16) niños y tres (3) padres, madres o representantes, a quienes se les aplicó técnicas como observación a la planificación docente, entrevista estructurada a los representantes a través de una guía de observación y un guion de entrevista. Estos instrumentos se validaron internamente, además, la información se analizó mediante la triangulación, pudiéndose elaborar un plan acción que fue ejecutado y evaluado. Dentro de las conclusiones se destaca que: la aplicación de estrategias lúdicas facilita el desarrollo cognitivo de los niños y niñas, así como sus habilidades lógicas, el uso de la estrategia de manipulación permite la motivación por ordenar y contar objetos. Igualmente, la aplicación de juegos de observación y percepción contribuyen al interés por conocer objetos, texturas y formas, en consecuencia, se genera una estimulación neuronal en los educandos.

Palabras clave: juego educativo; pensamiento; adaptación del estudiante.

Cómo citar este artículo:

Rocca, M. (2021). Experiencias Lúdicas en el Desarrollo del Pensamiento Lógico. Revista Scientific, 6(19), 208-227, e-ISSN: 2542-2987. Recuperado de: https://doi.org/10.29394/Scientific.issn.2542-2987.2021.6.19.10.208-227

Fecha de Recepción: 07-08-2020
Fecha de Aceptación:

21-12-2020
Fecha de Publicación: 05-02-2021 
OAI-PMH: http://www.indteca.com/ojs/index.php/Revista Scientific/oai

Artículo Original / Original Article

\title{
Playful Experiences in the Development of Logical Thought
}

\begin{abstract}
The purpose of the research was to implement playful experiences for the teaching of logical thinking in the Bolivarian Initial Education Center Misia Jacinta de Crespo, Corazón de Jesús parish of the Barinas municipality, Barinas state. Methodologically, it was based on the socio-critical paradigm under the action research method in the phases provided by Hurtado and Toro (2005), diagnosis, planning, execution and evaluation; the informants being two (2) teachers, sixteen (16) children and three (3) fathers, mothers or representatives, who were applied techniques such as observation of the teaching planning, structured interview with the representatives through an observation guide and an interview script. These instruments were internally validated, in addition, the information was analyzed through triangulation, being able to develop an action plan that was executed and evaluated. Among the conclusions, it is highlighted that: the application of playful strategies facilitates the cognitive development of boys and girls, as well as their logical abilities, the use of the manipulation strategy allows motivation to order and count objects. Likewise, the application of observation and perception games contribute to the interest in knowing objects, textures and shapes, consequently, a neuronal stimulation is generated in the students.
\end{abstract}

Keywords: educational game; thinking; student adjustment.

How to cite this article:

Rocca, M. (2021). Playful Experiences in the Development of Logical Thought. Revista Scientific, 6(19), 208-227, e-ISSN: 2542-2987. Recovered from: https://doi.org/10.29394/Scientific.issn.2542$\underline{2987.2021 .6 .19 .10 .208-227}$

Date Received: 07-08-2020
Date Acceptance:

21-12-2020
Date Publication: 05-02-2021 


\section{Introducción}

Los procesos educativos del hombre facilitan su existencia, la satisfacción de necesidades de coexistencia en una sociedad regida por valores, principios morales y éticos consagrados básicamente en el uso de su libertad, amor por su patria, igualdad, equidad, seguridad, derecho a la alimentación, a llevar una vida digna, cultivar su conocimiento, mediante una educación de pensamiento libre, genuina, comprometida con los valores culturales del pueblo, para ello, es necesario contar con un sistema educativo de gestión humanística, creativa e innovadora, asimismo, con docentes capaces de estimular la vocación por aprender y convivir, de allí, la importancia de los aprendizajes iníciales de los niños y niñas.

La educación inicial en Venezuela es de gran importancia en el sistema educativo venezolano, porque es allí donde se dan los primeros contactos del niño con el ambiente escolar y social, los cuales contribuyen para el pleno desarrollo integral. En este nivel el infante construye el conocimiento lógicomatemático, el cual está compuesto por un conjunto de conocimientos, capacidades, aptitudes y destrezas que configuran su estructura lógica.

Por tanto, es durante los primeros años de vida en el cual el desarrollo cognoscitivo de los niños alcanza progresos, en especial en el área de matemática. En opinión de Valecillos (2018a): "el aprendizaje en niños y niñas cursantes del Nivel Preescolar, referentes a contenidos matemáticos depende en gran parte del contexto, aunado a, las circunstancias ofrecidas por el docente para que surja este tipo de Pensamiento Lógico-Matemático" (pág. 222).

Por ello, es necesario incorporar actividades a través de la lúdica en las relaciones de aprendizaje desarrolladas al interior de las aulas de clase, con la finalidad de contribuir en la asimilación de conceptos abstractos como lo son los números, mediante experiencias. Vale decir, que los docentes de educación inicial dan continuidad a los aprendizajes adquiridos por los niños 
en el hogar con el apoyo de los adultos significativos, en especial los padres, madres o representantes a través de orientaciones didácticas, estrategias y actividades prácticas enmarcadas en un clima de flexibilidad, innovación, creatividad; pero, respetando la individualidad del infante.

Sin lugar a duda, esta tarea se ve complementada con la participación, integración y vinculación de los padres, madres y representantes en las relaciones de aprendizaje, de manera, el niño pueda sentirse en un ambiente de confianza para que se desarrolle en condiciones que brinden estabilidad emocional, cognitiva, social como afectiva, para alcanzar una enseñanza de calidad y articulada al entorno educativo.

Desde este visón, se busca vías idóneas para lograr no solo una adaptación del niño al contexto educativo, sino también una estimulación temprana mediante experiencias lúdicas que le brinden oportunidades de aprendizaje a los niños por medio del juego, en especial en el desarrollo del pensamiento lógico-matemático. En este sentido, el profesional educativo debe planificar actividades para el perfeccionamiento de los órganos de los sentidos, es decir las relacionadas con la percepción viso auditiva, permitiendo el reconocimiento como la diferenciación de colores, formas y sonidos.

En consecuencia, se debe incentivar acciones mancomunadas para involucrar a los actores educativos en experiencias lúdicas con la finalidad de potenciar el desarrollo evolutivo de los infantes a través de un trabajo pedagógico. Vale decir, que las acciones emprendidas por los docentes desde las estrategias de los juegos, facilitan la praxis educativa de los niños y niñas mediante la motivación de actividades que requieren del pensamiento lógico, por tanto, el desarrollo de actividades lúdicas incita a los educandos a divertirse en el desarrollo de problemas que están en su entorno, esto facilita la incorporación de los padres y representantes en la formación inicial de sus hijos.

Desde la óptica de Valecillos (2018b): cuando el docente de educación 
inicial "no demuestra afecto, el tipo de estrategia que implementa carece de emotividad e incentivo emocional, para que éstos aprendan de forma significativa a desarrollar destrezas a avanzada edad, en este caso, para el desarrollo del Pensamiento Lógico-Matemático” (pág. 223).

Por consiguiente, al introducir experiencias lúdicas a través de juegos, se logra en los infantes acentuar su funcionalidad, así como acciones conducentes hacia la participación, interacción e interrelación del niño con diversos materiales y con sus pares, ampliando por ende, el proceso de socialización iniciado en el hogar; además, construya conocimientos. De allí, las experiencias lúdicas favorecen en los niños la construcción de autoconfianza, autonomía y la formación de la personalidad, convirtiéndose en actividades conducentes a la adquisición de aprendizajes significativos; razón por la cual el docente dinamicen espacios como tiempos en el aula para su implementación.

Sin embargo, la realidad observada en la educación inicial en el estado Barinas, en especial el Centro de Educación Inicial Bolivariano Misia Jacinta de Crespo, parroquia Corazón de Jesús del municipio Barinas, institución educativa en la cual se vienen realizando esfuerzos para desarrollar relaciones de aprendizaje con la participación de padres, madres o representantes para el desarrollo integral del niño, haciendo énfasis en experiencias lúdicas para la mediación del pensamiento lógico-matemático. Pese a ello, durante la jornada diaria en el nivel maternal se evidencia escasas actividades haciendo uso del juego, pudiese ser por desconocimiento, desaciertos en el conocimiento evolutivo o desmotivación para percibir la importancia del aprendizaje de los infantes.

Adicionalmente, se aprecia una carencia de vinculación de los padres en el proceso educativo de los niños, hecho que obstaculiza la praxis del docente en el aula que le permita al infante potenciar cognoscitivamente nociones lógico-matemáticas relacionadas con números, series, agrupar 
objetos, entre otros. Ante esta situación, es necesario implementar experiencias lúdicas con números para la enseñanza del pensamiento lógico en el Centro de Educación Inicial Bolivariano Misia Jacinta de Crespo, parroquia Corazón de Jesús del municipio Barinas, estado Barinas.

En referencia con el contexto teórico, se tiene que la sustentación teórica de la investigación tuvo como propósito el establecimiento de las investigaciones previas, así como la fundamentación teórica relacionadas con el objeto de estudio, de manera, se sustente la problemática evidenciada en el Centro de Educación Inicial Bolivariano Misia Jacinta de Crespo, parroquia Corazón de Jesús del municipio Barinas, estado Barinas.

Indica Arias (2012): que el marco de referencia, se corresponde con "una revisión exhaustiva de las teorías más recientes que describen todo lo que se sabe o se ha investigado ya sobre el fenómeno o evento que tenemos que investigar" (pág. 25); lo cual implica la existencia de información teórica para fundamentar el proceso investigativo.

Al realizar una revisión de investigaciones relacionadas con el tema, consideradas como investigaciones previas, se destaca el trabajo realizado por Núñez y Zapata (2018): abordado bajo el paradigma cuantitativo, de naturaleza aplicativa o práctica, apoyada en una investigación descriptivacorrelacional. El autor citado concluye, que el desarrollo del pensamiento se fortalece a través de juegos entre alumnos utilizando materiales didácticos adecuados e innovadores para el desarrollo de las relaciones lógicomatemáticas en niños y niñas de 4 a 5 años, a partir de la identificación de las necesidades específicas de los niños.

Del mismo modo, se exploró el trabajo desarrollado por Beltrán (2016): se enmarco en el paradigma mixto (Cualitativo y Cuantitativo), tipo descriptivo, en el que se utilizó como método, el diagnóstico en la revisión de documentos. Se concluye que manejando la lúdica y la diversión se puede cautivar el interés de los niños por participar activamente en el desarrollo de las intervenciones 
pedagógicas, este escenario depende del compromiso y motivación por parte de directivos, docentes, padres de familia. Por tanto, se requiere una consecuencia natural de prácticas de carácter progresivo para fortalecer el aprendizaje significativo a través del tiempo.

El autor concluye que el uso de materiales adecuados para aplicar en el aula las operaciones matemáticas no son solamente aquellos utilizados habitualmente, sino que pueden utilizase diferentes recursos de secuencias lógicas, sino que se pueden utilizar diferentes recursos para el desarrollo del presente ámbito basados en las posibilidades actuales de tener a la mano diversos materiales y permitir que la creatividad de cada educadora inicial fluya libremente basándose en sus previos conocimientos.

Se puede apreciar en ambas investigaciones, la necesidad que el docente de educación inicial asuma las actividades formativas en el pensamiento lógico-matemático constituyen un compendio de acciones de aprendizajes fundamentadas en la comprensión básica y creativa de situaciones elementales que pueden ser resueltas por los niños y niñas mediante su iniciativa por el juego pragmático natural de su entorno familiar.

Por otra parte, al hacerse la revisión de los fundamentos teóricos que sustentan el proceso indagativo, este se direccionó hacia las experiencias lúdicas, las cuales son desarrolladas en el aula maternal con la finalidad de introducir al niño hacia aprendizajes en ambientes agradables, de manera atractiva, natural, pero con sentido para el desarrollo de habilidades cognoscitivas. Generando, resultados satisfactorios para el infante porque se convierten en seres curiosos, creativos, innovadores.

En este sentido, Carrasquel (2013): argumenta que existe un alto valor didáctico en el hecho de incorporar las actividades lúdicas en el aula de clase, ya que "a través del juego, el niño vive situaciones reales o ficticias, que lo preparan para desempeñar su rol en la sociedad. Le produce placer, diversión y por lo general, es sinónimo de alegría y de bienestar" (pág. 31). Lo planteado 
por el autor, se direcciona hacia el desarrollo de actitudes e interacciones que propician aprendizajes significativos, precisando para ello de una satisfacción de necesidades educativas como de conocimiento, así como seguridad afectiva y libertad de actuación en el niño. En razón de ello, las experiencias lúdicas en educación inicial, contribuyen con el mejoramiento socioemocional, además, afectivos del infante con apoyo de adultos significativos, en especial de padres, madres o representantes.

En referencia con el pensamiento lógico-matemático en el nivel maternal, éste es construido por el niño en relación directa con su entorno, dado que actividades como la asociación de operaciones como clasificación, seriación e inclusión de la posibilidad de reversibilidad del pensamiento son necesarias en la construcción del concepto de número. De acuerdo con Alsina y León (2016a): "Ios niños van desarrollando su pensamiento matemático gracias a las interacciones constantes con las personas que les rodean y el entorno" (pág. 35); las cuales proporcionan herramientas necesarias para su adquisición, mediante la curiosidad presente en el medio sociocultural.

Por tanto, este tipo de conocimiento se adquiere de manera informal a través de experiencias numéricas asociadas con el juego, como indicar la edad con los dedos, poner velas en un pastel, comprensión de algunos cuantificadores como mucho, poco, algunos, identificación del tiempo (día, noche), entre otros, las cuales sirven de fundamento para un aprendizaje formal, incluyendo la comparación y observación de cambios o transformaciones.

En consecuencia, la interiorización del pensamiento lógico-matemático en niños de educación inicial, se logra a través de experiencias lúdicas, es decir, a través del juego se alcanza un desarrollo socio emocional afectivo equilibrado, además, es innegable que en los contextos escolares se puedan lograr estas estrategias para el proceso de aprendizaje. Este planteamiento, es congruente con los aportes de Lugo, Vilchez y Romero (2019): señalan que 
"en el proceso de aprendizaje, los conceptos lógico-matemáticos constituyen un instrumento fundamental y útil, porque a través de estos los niños expresan cada día sus conocimientos en cada una de las experiencias de formación educativa" (pág. 19).

De este modo, se estaría desarrollando habilidades para el pensamiento lógico-matemático en los niños y niñas de educación inicial, en ese sentido, es significativo la integración de los padres, madres o representantes quienes reforzarán los aprendizajes alcanzados en el hogar.

Atendiendo a ello, el estudio tuvo como objetivo general: Implementar experiencias lúdicas para la enseñanza del pensamiento lógico en el Centro de Educación Inicial Bolivariano Misia Jacinta de Crespo, parroquia Corazón de Jesús del municipio Barinas, estado Barinas, Venezuela. Asimismo, como objetivos específicos se consideró: Diagnosticar las actividades aplicadas por el docente para la enseñanza del pensamiento lógico-matemático en educación inicial; Describir las actividades realizadas por los niños para el aprendizaje del pensamiento lógico-matemático en educación inicial; Diseñar experiencias lúdicas para el desarrollo del pensamiento del lógico-matemático en el Centro de Educación Inicial Bolivariano Misia Jacinta de Crespo; Ejecutar experiencias lúdicas para el desarrollo del pensamiento lógico-matemático en el Centro de Educación Inicial en referencia; y Evaluar logros alcanzados en la implementación de experiencias lúdicas para el desarrollo del pensamiento lógico-matemático en el Centro de Educación Inicial en estudio.

\section{Metodología}

El proceso investigativo requirió asumir una posición metodológica para contribuir en la generación de conocimientos; por tal razón, la autora de la indagación consideró ubicarse en el paradigma cualitativo, de acuerdo con Gil, León y Morales (2017): este modelo "centra su estudio en los significados de las acciones humanas y la vida social, en medio de una realidad dinámica, 
múltiple y holística" (pág. 73). En ese sentido, el estudio busco la interpretación de la realidad en que se desenvuelven los docentes con el objeto de interpreta y comprender su comportamiento y proponer soluciones coherentes y acertadas a su praxis educativa

En relación con método, se estima el más conveniente fue el de investigación acción, porque se involucró a los actores educativos (docentes, niños, padres, madres y representantes) en la realidad estudiada, logrando con ello, una transformación a partir de la reflexión de las experiencias educativas.

Para lograr con éxito el desarrollo de la indagación se procedió a implementarse las fases de la investigación-acción, según Murcia (1990), citado por Hurtado y Toro (2005): la cual se describen como "10 fase exploratoria de la comunidad e identificación de necesidades básicas; $2^{\circ}$ descripción de la problemática con sus variables e indicadores de Valoración; $3^{\circ}$ elaboración de una estrategia educativa con sus acciones de ejecución" (pág. 186-187). En efecto, la investigación acción participativa no es rígida en cuanto a las fases para lograr los objetivos puesto que se trata de un proceso muy dinámico.

Por consiguiente, se realizó un reconocimiento de la realidad mediante la aplicación de técnicas como la observación a la planificación docente, así como la entrevista a profundidad, e instrumentos de recolección de información derivados de éstas: Guía de observación y guion de entrevista, aplicados a dos (2) docentes, tres (3) representantes y dieciséis (16) niños; los cuales fueron analizados mediante un proceso de categorización, contrastación, triangulación y teorización de los hallazgos; para posteriormente, elaborar una planificación a través de un plan acción que fue ejecutado y evaluado.

Con el objeto de darle mayor validez a la investigación los instrumentos de recolección de información se sometieron a una revisión de expertos en el 
área metodológica y educativa, asimismo, se desarrolló el proceso de teorización mediante el análisis de los resultados del diagnóstico en contraste con los conceptos considerados por autores reconocidos descritos en las bases teóricas consultadas.

\section{Resultados (análisis e interpretación de los hallazgos)}

Esta fase estuvo direccionada hacia el análisis de situaciones reales en el entorno educativo, con la finalidad de detectar problemas en la institución educativa objeto de estudio, previo establecimiento de la unidad de análisis, categorías y subcategorías, como se evidencia en la tabla 1.

Tabla 1. Categorización.

\begin{tabular}{|c|c|c|c|}
\hline $\begin{array}{c}\text { Unidad de } \\
\text { Análisis }\end{array}$ & Categorías & Subcategoría & Preguntas \\
\hline \multirow{4}{*}{$\begin{array}{l}\text { Pensamiento } \\
\text { lógico-matemático } \\
\text { con números en } \\
\text { educación inicial }\end{array}$} & $\begin{array}{l}\text { Cualidades } \\
\text { sensoriales }\end{array}$ & $\begin{array}{l}\text { 1a.- Características sensoriales } \\
\text { 1b.- Agrupaciones } \\
\text { 1c.- Clasificaciones }\end{array}$ & $\begin{array}{l}1 \\
2 \\
3\end{array}$ \\
\hline & Cantidades & $\begin{array}{l}\text { 2a.- Cuantificadores } \\
\text { 2b.- Inicio del conteo } \\
\text { 2c.- Correspondencias cuantitativas }\end{array}$ & $\begin{array}{l}4 \\
5 \\
6\end{array}$ \\
\hline & $\begin{array}{l}\text { Posiciones y } \\
\text { Formas }\end{array}$ & $\begin{array}{l}\text { 3a.- Posición relativa } \\
\text { 3b.- Construcción de líneas }\end{array}$ & $\begin{array}{l}7 \\
8\end{array}$ \\
\hline & $\begin{array}{c}\text { Atributos } \\
\text { mesurables }\end{array}$ & $\begin{array}{l}\text { 4a.- Magnitudes } \\
\text { 4b.- Tamaños }\end{array}$ & $\begin{array}{c}9 \\
10\end{array}$ \\
\hline
\end{tabular}

Fuente: La Autora (2019).

Durante el transcurso de la investigación acción, fue necesario conocer la realidad estudiada, reflexionarla de manera se pudiera generar una transformación, relacionada con las experiencias lúdicas con números para la enseñanza del pensamiento lógico en Centro de Educación Inicial Bolivariano Misia Jacinta de Crespo, parroquia Corazón de Jesús del municipio Barinas, estado Barinas. En función de ello, se aplicó una guía de observación, así como un guion de entrevista a los informantes previamente identificados que a efectos del presente artículo estuvo contentiva de una pregunta por 
categoría, siendo la información que fue recogida en una matriz, como se presenta en la tabla 2.

Tabla 2. Guía de Observación a la Planificación Docente.

\begin{tabular}{|c|l|c|c|}
\hline N. $^{\circ}$ & \multicolumn{1}{|c|}{ El docente: } & Si & No \\
\hline 1 & $\begin{array}{l}\text { Incluye actividades para que el niño reconozca características sensoriales } \\
\text { de los objetos. }\end{array}$ & $\mathrm{x}$ & \\
\hline 2 & Introduce al niño en el uso de cuantificadores (muchos, pocos, algunos). & & $\mathrm{X}$ \\
\hline 3 & Incorpora actividades para que el niño construya líneas. & $\mathrm{X}$ & \\
\hline 4 & $\begin{array}{l}\text { Aplica actividades para que el niño use de forma comprensible términos } \\
\text { como largo o corto. }\end{array}$ & $\mathrm{x}$ \\
\hline
\end{tabular}

Fuente: La Autora (2019).

La guía de observación aplicada a la planificación docente, refleja este profesional incluye actividades relacionadas con las cualidades sensoriales de los objetos; pero, no introduce en la jornada diaria cuantificadores empleando términos como muchos, pocos, todos, algunos. Del mismo modo, se pudo evidenciar, incluye actividades la construcción de líneas tanto rectas como curvas; además, no aplica actividades para que el infante use de forma comprensible términos como largo o corto, como se muestra en la tabla 3.

Tabla 3. Observación aplicada a los Niños.

\begin{tabular}{|c|l|c|c|}
\hline N. $^{\mathbf{0}}$ & \multicolumn{1}{|c|}{ El niño: } & Si & No \\
\hline 1 & Reconoce características sensoriales de los objetos. & 4 & 12 \\
\hline 2 & Emplea cuantificadores como muchos, pocos, algunos. & 6 & 10 \\
\hline 3 & Construye líneas rectas o curvas. & 14 & 2 \\
\hline 4 & Utiliza de forma comprensible términos como largo o corto. & 6 & 10 \\
\hline
\end{tabular}

Fuente: La Autora (2019).

Los resultados obtenidos de la aplicación de la guía de observación a los infantes, la misma, reflejan que doce (12) niños no reconocen características sensoriales de los objetos y cuatro (4) si lo hacen, asimismo, diez (10) no emplean cuantificadores como mucho, pocos, algunos; seis (6) si lo aprovechan y diez (10) no utilizan comprensiblemente términos como largo o corto, mientras seis (6) si los manejan, aun cuando catorce (14) construye líneas rectas o curvas y dos (2) no lo hacen. Estos resultados son 
metodológicamente contrastados con el guion de entrevista administrado a los docentes. Para ampliar la información se presenta a continuación la tabla 4.

Tabla 4. Guion de Entrevista aplicado a los Docentes.

\begin{tabular}{|c|c|c|c|}
\hline N. ${ }^{-}$ & Pregunta & Categoría & $\begin{array}{rr}\text { Respuesta } \\
\end{array}$ \\
\hline 1 & $\begin{array}{l}\text { ¿Qué actividades puede } \\
\text { incluir en su } \\
\text { planificación para que } \\
\text { niño identifique } \\
\text { cualidades sensoriales? }\end{array}$ & $\begin{array}{l}\text { Cualidades } \\
\text { sensoriales }\end{array}$ & $\begin{array}{l}\text { D1: Mi visión es que se pueden introducir } \\
\text { actividades en las cuales el niño pueda } \\
\text { identificar progresivamente cualidades } \\
\text { sensoriales, por ejemplo, a través de } \\
\text { texturas; pero no ubico las mismas. } \\
\text { D2: A través de objetos con diferentes } \\
\text { texturas, tamaños, colores, de manera que } \\
\text { pueda agrupar, clasificar, seriar. }\end{array}$ \\
\hline 2 & $\begin{array}{l}\text { ¿De qué manera } \\
\text { introduce los } \\
\text { cuantificadores en el } \\
\text { aula maternal? }\end{array}$ & Cantidades & $\begin{array}{l}\text { D1: En explicaciones comprensibles para } \\
\text { el niño, en los cuales les muestre que son } \\
\text { pocos, muchos; pero no cuales son. } \\
\text { D2: Cuando realizamos actividades de la } \\
\text { rutina diaria, les manifiesto que somos } \\
\text { muchos en el aula, pocos vinieron a clase } \\
\text { y así sucesivamente. }\end{array}$ \\
\hline 3 & $\begin{array}{l}\text { ¿Qué contenidos } \\
\text { pueden ser } \\
\text { desarrollados para que } \\
\text { el niño emplee las } \\
\text { posiciones y formas en } \\
\text { el aula maternal? }\end{array}$ & $\begin{array}{l}\text { Posiciones } \\
\text { y Formas }\end{array}$ & $\begin{array}{l}\text { D1: La mayoría de los contenidos, pero no } \\
\text { sé cómo hacerlo. } \\
\text { D2: En especial en aquellos que se pueda } \\
\text { verbalizar posiciones relativas; pero no } \\
\text { logro ubicar las actividades. }\end{array}$ \\
\hline 4 & $\begin{array}{c}\text { ¿De qué manera incluye } \\
\text { contenidos relacionados } \\
\text { con magnitudes en el } \\
\text { aula maternal? }\end{array}$ & $\begin{array}{l}\text { Atributos } \\
\text { mesurables }\end{array}$ & $\begin{array}{l}\text { D1: En aquellos donde se puedan mediar } \\
\text { actividades asociadas a magnitudes de } \\
\text { longitud como largo, ancho; aunque no sé } \\
\text { qué actividades planificar. } \\
\text { D2: En actividades para comprender el } \\
\text { tamaño de los objetos; pero es difícil } \\
\text { planificar este tipo de acciones. }\end{array}$ \\
\hline
\end{tabular}

Fuente: La Autora (2019).

En la información recolectada en el guion de entrevista, se aprecian las opiniones emitidas por los docentes sobre las actividades aplicadas en el aula, durante la enseñanza del pensamiento lógico-matemático en el nivel maternal. Se evidencia que los docentes aplican pocas estrategias relacionados con las acciones de diseño de actividades didácticas para el desarrollo cognitivo del niño; además, al realizar la observación, se evidencia la carencia de experiencias lúdicas que contribuyan con el proceso para la adquisición de las 
nociones lógico-matemáticas en educación inicial. Con respecto a lo antes mencionado, se comparte opinión con Alsina y León (2016b): al señalar que "se ha podido comprobar que cuánto más pequeños son los niños, más reducido es el número de acciones que realizan, mientras que a medida que van creciendo el número de acciones se va incrementando" (pág. 59); haciéndose necesario una planificación con experiencias lúdicas.

\subsection{Planificación, Ejecución y Evaluación}

En este apartado se refleja el proceso de planificación, ejecución y evaluación de las experiencias lúdicas, previa considerando, a estas como parte de las fases de la investigación cualitativa; con el propósito de transformar la práctica pedagógica desarrollada por los docentes en el nivel maternal, específicamente en la enseñanza del pensamiento lógicomatemático.

En relación con la planificación se destaca que fue originada bajo la sistematización de los pensamientos reflejados por los docentes objeto de estudio, lo cual fue traducido como un diagnostico genuino real de la problemática presentada en el Centro de Educación Inicial Bolivariano Misia Jacinta de Crespo, parroquia Corazón de Jesús del municipio Barinas, estado Barinas.

En cuanto a la ejecución de las actividades programadas, se desarrollaron bajo un clima de cordialidad, entusiasmo por parte de los docentes, padres y representantes los cuales se mostraron su interés por participar activamente en cada una de las actividades planificadas, facilitando el intercambio de experiencia y la construcción colectiva de nuevos recursos lúdicos. Vale decir, que la mayoría de los niños logró realizar y manejar adecuadamente las instrucciones del docente al momento de incorporarse a los juegos didácticos, allí fue fundamental la generación de una comunicación asertiva promoviendo la confianza y el estímulo de conocer o explora. Parte 
de esta experiencia es descrita a continuación en la tabla 5.

Tabla 5. Experiencia Lúdica.

\begin{tabular}{|c|c|c|c|}
\hline $\begin{array}{l}\text { Finalidad: Bri } \\
\text { a mediación } \\
\text { Jbjetos para }\end{array}$ & riencia & ncimi & $\begin{array}{l}\text { padres, madres y representantes para } \\
\text { las características sensoriales de los } \\
\text { ón, correspondencias cualitativas y }\end{array}$ \\
\hline $\begin{array}{l}\text { Cont } \\
\text { - Características } \\
\text { - Agrupación } \\
\text { - Clasificación } \\
\text { - Correspondenc } \\
\text { - Seriaciones } \\
\text { Estra } \\
\text { Manipu }\end{array}$ & $\begin{array}{l}\text { o } \\
\text { isoriales } \\
\text { cualitativas } \\
\text { a } \\
\text { ión }\end{array}$ & $\begin{array}{l}\text { - Saludo de b } \\
\text { - Realización } \\
\text { - Orientacion } \\
\text { desarrollo de } \\
\text { a docentes, p } \\
\text { - Conformaci } \\
\text { conclusiones } \\
\text { - Plenaria par } \\
\text { - Realimentac }\end{array}$ & $\begin{array}{l}\text { Actividades } \\
\text { nvenida por parte de la investigadora. } \\
\text { dinámica. } \\
\text { sobre las experiencias lúdicas en el } \\
\text { ensamiento lógico en el Nivel Maternal } \\
\text { Ires, madres y representantes. } \\
\text { de equipos de trabajo para realizar } \\
\text { la lectura de conclusiones. } \\
\text { n y evaluación de la actividad. }\end{array}$ \\
\hline Facilitador & $\begin{array}{l}\text { Tiempo de } \\
\text { ejecución }\end{array}$ & Fecha & Valoración \\
\hline $\begin{array}{l}\text { Docente } \\
\text { investigador } \\
\text { Especialista }\end{array}$ & $\begin{array}{c}\text { Tres Horas } \\
\text { teórico- } \\
\text { prácticas }\end{array}$ & $19 / 10 / 2018$ & $\begin{array}{l}\text { Asistencia y participación. } \\
\text { Observación al participante. } \\
\text { Aportes a la discusión. } \\
\text { Acuerdos grupales. }\end{array}$ \\
\hline
\end{tabular}

Fuente: La Autora (2019).

Las acciones pedagógicas programadas, se efectuaron el día viernes 19 de octubre del año 2018, en las instalaciones del Centro de Educación Inicial Bolivariano Misia Jacinta de Crespo, parroquia Corazón de Jesús del municipio Barinas, estado Barinas, Venezuela, con la finalidad de sensibilizar mediante un círculo de formación para brindar experiencias Lúdicas a docentes, padres, madres y representantes para la mediación de actividades de reconocimiento de las características sensoriales de los objetos para su posterior agrupación, clasificación, correspondencias cualitativas y seriaciones en los niños de Educación Inicial.

La investigadora inicia la actividad dando un saludo a todos los participantes, seguidamente relata la metodología de trabajo señalando el objetivo de la dinámica de presentación denominada ¿Quién soy yo?, al cual consistió en decir sus nombre y apellido aludiendo a una figura de un objeto $o$ 
animal. Una vez culminada la actividad se le pregunto a los docentes su opinión de la dinámica y su impacto en el clima del grupo, los docentes respondieron: Sí, porque es una forma de introducir la motivación a las actividades formativas, haciendo uso del juego. Inmediatamente, la investigadora procede a dar inicio al círculo de formación, señalando el desarrollo del pensamiento lógico-matemático es un factor clave en el desarrollo cognitivo del niño, porque éste crea relaciones entre los objetos manipulándolos, estableciendo características.

Por tanto, el niño va construyendo un conocimiento global articulado con lo motriz e intelectual mediante la realización de actividades a través de la observación, percepción, tocar, sentir, entre otras; inmediatamente, la investigadora, fue entregando a los participantes diferentes objetos con diferentes texturas, colores, formas.

Al culminar la intervención de la investigadora, requiere a los docentes conformen equipos de trabajo para la lectura del material sobre el tema; además de la realización de ejercicios prácticos con los objetos entregados previamente para que estos reconozcan texturas, agrupación por color, clasificación, correspondencia, seriación, derivándose conclusiones, las cuales fueron expuestas en una plenaria. Se realimentó la actividad a través de preguntas, respondidas satisfactoriamente, para proceder a la toma de notas en el registro de incidencias llevado por la docente investigadora, quien empleó la información para valorar la realización del círculo de formación.

Sobre el proceso valorativo, se expone el acuerdo colectivo de incorporar un compendio de actividades lúdicas en la planificación del docente, asimismo, el diseño de acciones formativas en el contexto pedagógico de juegos matemáticos que faciliten la incorporación de los padres y presentantes en la formación de sus hijos, de igual forma, se apreció que los docentes están dispuesto a emplear actividades lúdicas relacionadas con situaciones en la que interviene el color, así como otras cualidades sensoriales como la textura, 
formas de objetos para su reconocimiento, clasificación y seriación.

\section{Conclusiones}

Los resultados en el devenir investigativo se logró la implementación de experiencias lúdicas en el desarrollo del pensamiento lógico en el Centro de Educación Inicial Bolivariano Misia Jacinta de Crespo, parroquia Corazón de Jesús del municipio Barinas, estado Barinas, así como desarrollar todos y cada uno de los propósitos de la investigación. A través del diagnóstico, se pudo determinar las actividades aplicadas por el docente para la enseñanza del pensamiento lógico-matemático en el nivel maternal, para planificar actividades para la transformación de esta, con la participación de docentes, padres madres y representantes, las cuales fueron ejecutadas y evaluadas en su totalidad, demostrando son actividades de fácil aplicación en el referido nivel.

La aplicación de estrategia lúdica facilita el desarrollo cognitivo de los niños y niñas, así como sus habilidades lógicas, el uso de la estrategia de manipulación permite la motivación por ordenar y contar objetos. Igualmente, la aplicación de juegos de observación y percepción contribuyen al interés por conocer objetos, texturas y formas, en consecuencia, se genera una estimulación neuronal en los estudiantes, lo cual es significativo en el proceso de aprendizaje educativo.

Debido a estos hallazgos, se denota el alto significado educativo que tiene para los niños y niñas el desarrollo de actividades lúdicas que faciliten su disposición a incorporarse en tareas de resolución de situaciones sin temores. En ese sentido, durante la planificación del docente, pueden incluirse actividades lúdicas dirigidas a mejorar los aspectos cognitivos, corporales y motrices, asimismo, se sugiere a los docentes objeto de estudio la investigación, planificación, análisis y aplicación de actividades lúdicas especificas en función de la naturaleza de su matrícula de niños y niñas. 
De igual forma, se sugiere continuar con una planificación de experiencias lúdicas, las cuales favorecen el desarrollo del pensamiento lógico de los niños del nivel maternal, en los cuales se involucren padres, madres y representantes, posibilitando, de esta manera, la apertura de espacios participativos que vinculen las relaciones de aprendizaje tanto en el Centro de Educación Inicial como el hogar.

\section{Referencias}

Alsina, Á., \& León, N. (2016a,b). Acciones Matemáticas de 0 a 3 años a partir de Instalaciones Artísticas. Educatio Siglo XXI, 34(2), 33-62, ISSN: 1699-2105; e-ISSN: 1989-466X. Recuperado de:

\section{http://dx.doi.org/10.6018/j/263801}

Arias, F. (2012). El Proyecto de Investigación: Introducción a la Metodología Científica. 6ta edición, ISBN: 980-07-8529-9. Caracas, Venezuela: Editorial Episteme.

Beltrán, C. (2016). Guía de Material Didáctico Innovador para el Aprendizaje del Ámbito de las Relaciones Lógico-Matemáticas en Niños y Niñas de 4 A 5 años del Centro de Educación Inicial "Miss Travesuritas", Ciudad de Quito. Tesis. Quito, Ecuador: Universidad Tecnológica Equinoccial. Recuperado de:

http://repositorio.ute.edu.ec/bitstream/123456789/15420/1/66816 1.pd $\underline{f}$

Carrasquel, D. (2013). Estrategias Basadas en el Eje Lúdico para Lograr la Articulación Efectiva entre la Educación Inicial y la Educación Primaria. Trabajo de grado. Caracas, Venezuela: Universidad Central de Venezuela. Recuperado de:

http://saber.ucv.ve/bitstream/123456789/17159/1/COMPLETA.pdf

Gil, J., León, J., \& Morales, M. (2017). Los paradigmas de investigación educativa, desde una perspectiva crítica. Revista Conrado, 13(58), 
72-74, ISSN: 2519-7320; e-ISSN: 1990-8644. Recuperado de:

https://conrado.ucf.edu.cu/index.php/conrado/article/view/476

Hurtado, I., \& Toro, J. (2005). Paradigmas y Métodos de la Investigación en

Tiempos de Cambio. 5ta Edición, ISBN: 980-328-413-4. Valencia,

Carabobo, Venezuela: Episteme Consultores Asociados, C.A.

Lugo, J., Vilchez, O., \& Romero, L. (2019). Didáctica y desarrollo del pensamiento lógico matemático. Un abordaje hermenéutico desde el escenario de la educación inicial. Revista Logos Ciencia \& Tecnología, 11(3), 18-29, ISSN: 2145-549X; e-ISSN: 2422-4200. Recuperado de: https://doi.org/10.22335/rlct.v11i3.991

Núñez, A., \& Zapata, M. (2018). Desarrollo del Pensamiento Matemático a Través de Juegos en Alumnos del Nivel Inicial en la Institución Educativa Particular Santa María Reina de Lima Norte - Comas 2015. Tesis. Huacho, Perú: Universidad Nacional José Faustino Sánchez Carrión. Recuperado de:

http://repositorio.unifsc.edu.pe/bitstream/handle/UNJFSC/3314/NU\%C 3\%91EZ\%20CABALLERO\%20y\%20ZAPATA\%20RODRIGUEZ.pdf Valecillos, B. (2019a,b). Desde la Pedagogía de la Ternura: Inicio de lo Lógico-Matemático en Preescolar. Revista Scientific, 4(12), 220-239, e-ISSN: 2542-2987. Recuperado de:

https://doi.org/10.29394/Scientific.issn.2542-2987.2019.4.12.11.220$\underline{239}$ 


\section{María Nazareth Rocca Báez}

e-mail: nazarethrocca2017@gmail.com

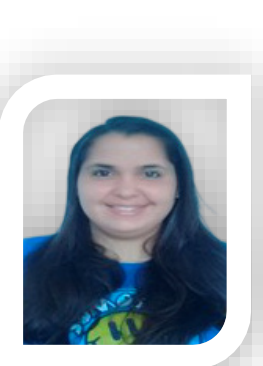

Nacida en Valencia, Venezuela, el 7 de marzo del año 1988. Egresada como profesora en Educación Inicial de la Universidad Pedagógica Experimental Libertador (UPEL-IMPM, 2015), extensión académica Barinas; además, me desempeño como docente de aula en el Centro de Educación Inicial Bolivariano Misia Jacinta de Crespo, parroquia Corazón de Jesús del municipio Barinas, estado Barinas, Venezuela. 